\title{
Auditory Feedback in Music Performance: The Role of Melodic Structure and Musical Skill
}

\author{
Peter Q. Pfordresher \\ University of Texas at San Antonio
}

\begin{abstract}
Five experiments explored whether fluency in musical sequence production relies on matches between the contents of auditory feedback and the planned outcomes of actions. Participants performed short melodies from memory on a keyboard while musical pitches that sounded in synchrony with each keypress (feedback contents) were altered. Results indicated that altering pitch contents can disrupt production, but only when altered pitches form a sequence that is structurally similar to the planned sequence. These experiments also addressed the role of musical skill: Experiments 1 and 3 included trained pianists; other experiments included participants with little or no musical training. Results were similar across both groups with respect to the disruptive effects of auditory feedback manipulations. These results support the idea that a common hierarchical representation guides sequences of actions and the perception of event sequences and that this coordination is not acquired from learned associations formed by musical skill acquisition.
\end{abstract}

Keywords: sequence production, music performance, auditory feedback, skill acquisition

The actions involved in sequential behaviors such as singing, playing the piano, or speaking create sounds called auditory feedback. Researchers typically explore the degree to which fluent production relies on auditory feedback by manipulating the relations between actions and feedback (the altered auditory feedback paradigm). Until recently, most investigations have focused on temporal synchrony between perception and action, the necessity of which is demonstrated by the disruptive effect of delayed auditory feedback (DAF; first demonstrated in speech by Black, 1951; and Lee, 1950; and in music by Havlicek, 1968), in which feedback events are asynchronous with actions. However, more recent evidence suggests that production of musical sequences can be disrupted when pitch events (feedback contents) are synchronous with keypresses but are altered to match a pitch intended for a past (Pfordresher, 2003a) or future (Pfordresher \& Palmer, in press) sequence position. This article addresses the role of feedback contents in music performance by manipulating similarity of the structure formed by sequences of feedback events to the planned sequence. Planned events comprise pitches that would typically result from a planned action (i.e., on an unaltered keyboard), whereas perceived events comprise auditory feedback events.

This material is based on work supported in part by National Science Foundation Grant BCS-0344892 and by a University of Texas at San Antonio faculty research award. Portions of these results were presented at the meeting of the Psychonomic Society, November 2004, Minneapolis, Minnesota. Erik M. Gallemore and Augusto Petacchi ran the experiments. I thank Caroline Palmer, Bruno Repp, and Mari Riess Jones for comments on an earlier version and Brian Benitez, Julliann Canady, and Erik Gallemore for help in preparing the manuscript.

Correspondence concerning this article should be addressed to Peter Q. Pfordresher, Department of Psychology and Institute for Music Research, University of Texas at San Antonio, 6900 North Loop 1604 West, San Antonio, TX 78249-0652. E-mail: peter.pfordresher@utsa.edu
A second issue that this article addresses has to do with the influence of acquired musical skill on the use of auditory feedback in music performance. It is intuitive to propose that associations between actions and pitches will result from training on the keyboard. However, it is possible that people are sensitive to relations between planned movement trajectories and the concurrent pattern of sequential pitch movements regardless of past experience. The current research tests these alternative possibilities by exposing individuals with and without formal piano training to similar altered feedback conditions during musical keyboard production tasks.

\section{Effects of Altered Auditory Feedback}

Some previous results have suggested that disruption from altered feedback occurs only when sounds and actions are synchronous (as in DAF) and that negligible disruption results from manipulations of feedback contents. A qualification of these results is that nondisruptive manipulations of feedback contents result in feedback pitches that are unrelated to planned pitches. For instance, Finney (1997) failed to find disruption of piano performance from a quasi-random mapping between piano keys and pitches. Howell and Archer (1984) likewise demonstrated that DAF disruption of speech was not qualitatively altered when feedback contents (phonemes) were converted to a square wave tone. Other results indicate that piano performance is not disrupted when auditory feedback is removed (Finney, 1997; Finney \& Palmer, 2003; Gates \& Bradshaw, 1974; Repp, 1999). Such findings have led to the idea that fluent production relies on synchronization between perception and action but not on matches between planned and perceived event contents (e.g., Howell, 2001, 2004; Howell \& Archer, 1984; Howell \& Au-Yeung, 2002; Howell, Powell, \& Khan, 1983). Lack of disruption from altered contents likewise disconfirms closed-loop accounts (e.g., Chase, 1965; Fairbanks, 1954; Lee, 1950; see also Adams, 1971; Wiener, 
1948), which predict disruption from virtually any change of auditory feedback.

Recent results from music performance, however, have demonstrated that certain alterations of feedback contents can disrupt production. In particular, serial shifts, which present pitch events intended for past or future sequence positions in synchrony with each keypress, disrupt production, primarily by increasing error rates (Pfordresher, 2003a; Pfordresher \& Palmer, in press; see Stöcker, Sebald, \& Hoffman, 2003, for a similar manipulation in serial response tasks). For instance, a serial shift of lag 1 would result in the performer hearing the pitch typically associated with the most recently produced action at each keypress. Given that serial shifts maintain synchrony between an action and the altered auditory feedback, disruption must occur because planned and perceived outcomes of actions differ and is unlikely to reflect disruption of low-level timing mechanisms that regulate synchrony.

\section{Auditory Feedback and Planning}

How are these differing results to be resolved? Planning actions and perceiving the consequences of actions may be guided by the same representation of sequence structure (Hommel, Müsseler, Aschersleben, \& Prinz, 2001; MacKay, 1987; Müsseler, 1999; Prinz, 1997). Figure 1 depicts this assumption in a format similar to that used by Hommel et al. (2001). A common representation of sequence events guides the production of the current event (E5; letter-number combinations indicate the pitch categories produced in a sequence) and the perceived result. According to this framework, events are produced and/or perceived to the extent that they are activated over time (cf. MacKay, 1987; Palmer \& Pfordresher, 2003). ${ }^{1}$ Because perception and action share a common representation, serial shifts may result in mismatches between activation from perception and activations generated by planning of actions, resulting in persistent interference. By contrast, the randomly selected pitch will match the planned event only rarely (i.e., by chance) and thus should cause negligible interference. Normal auditory feedback adds confirmatory activation to the current event but is not used to trigger retrieval of the next event.

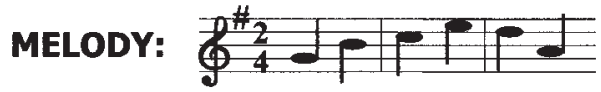

\section{SHARED REPRESENTATION:}

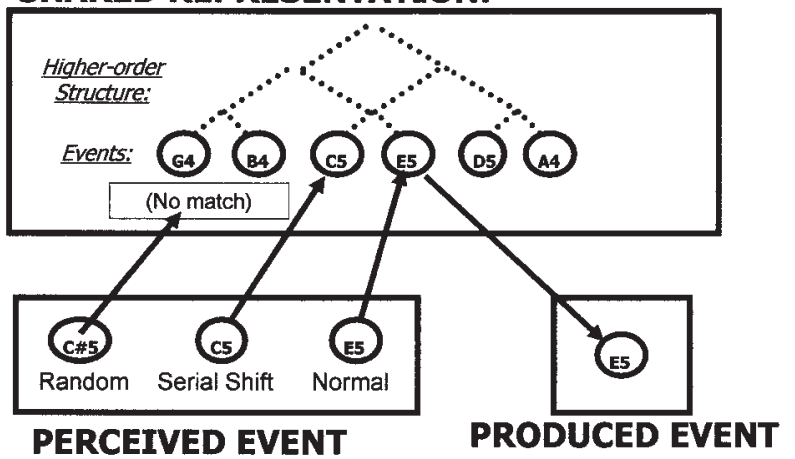

Figure 1. Schematic depiction of the proposed theoretical framework and how it accounts for disruption from altered feedback contents.
A second assumption of this framework is that events in the shared representation are incorporated within a higher order structure that determines serial order, here shown as a hierarchical tree (cf. Collard \& Povel, 1982; Jones, 1976; Martin, 1972; Rosenbaum, Kenny, \& Derr, 1983). Thus, the shared representation can be considered a two-tiered framework comprising both individual events and their organization. This framework predicts that performers may be sensitive not only to zero-order matches between perceived and planned events but also to matches between the global organization of perceived events and the planned action sequence. For instance, altered feedback that results in a permutation of the planned sequence generates a feedback sequence in which individual pitches match pitches intended for production at other positions, but the global organization of the feedback sequence may bear no relation to the organization of the planned sequence. The current research focuses on one characteristic of structure, serial order, as a starting point, although more finegrained characteristics of structural relations (e.g., melodic contour) are currently being explored (Pfordresher, 2005).

\section{Current Experiments}

Five experiments were designed to test the influence of altered feedback pitch contents on music performance. In all experiments, participants performed short musical sequences from memory while listening to auditory feedback over headphones. During certain performances, the pitch contents resulting from each produced action were altered in various ways to produce sequences of feedback events that varied with respect to their similarity to the planned sequence. An additional sixth experiment addressed sensitivity to perception-action relations in nonpianists by having participants rate the similarity of the perceived sequence to the expected results of actions.

One goal of the present experiments is to confirm that serial shifts disrupt production, in contrast with the negligible disruption that results from randomized pitches or feedback absence (Finney, 1997; Pfordresher, 2003a; Repp, 1999). Experiments 1 and 2 include these conditions in the same session with pianists and nonpianists, respectively. Experiments 3-5 further test the role of perception-action similarity by varying the similarity of altered auditory feedback sequences to the planned sequence. The general prediction for these experiments is that disruption will scale with similarity. Maximal disruption should be found for serial shifts, in which sequence structure is identical but the sequential coordination of perception and action is altered. For other sequences that are not structurally identical to the planned sequence, more disruption should be found for feedback sequences that are structurally similar to the planned sequence, relative to sequences that are structurally dissimilar.

A second goal of these experiments is to test whether disruption from altered feedback contents results from musical training. Clearly, musical training on the keyboard generates learned associations between actions and resulting pitches. Recent evidence

\footnotetext{
${ }^{1} \mathrm{I}$ adopt the term activation in a generic sense, to refer to variations in the prominence of sequence events across time. Activation mechanisms figure into many models of sequence production (e.g., Dell, 1986). By way of comparison, my use of the term activation is similar to MacKay's (1987) use of the term priming.
} 
indicates that musical training enhances action-effect associations (Drost, Rieger, Brass, Gunter, \& Prinz, 2005; Keller \& Koch, 2005). Thus, nonpianists may show less disruption than do pianists when auditory feedback pitches are altered. However, it is also possible that the coordination of perception and action operates on a domain-general level, resulting in similar patterns of disruption regardless of skill. All persons, regardless of training, may be sensitive to correlations between movement trajectories and the trajectories formed by changes in pitch direction.

\section{Experiment 1}

In Experiment 1, trained pianists performed short, unfamiliar melodies from memory while listening to auditory feedback over headphones. Trials followed a synchronization-continuation paradigm (Stevens, 1886; Wing \& Kristofferson, 1973): Participants produced the sequence with normal feedback, in synchrony with a metronome, during the synchronization phase and then continued to perform without a metronome during the continuation phase. Auditory feedback during continuation could be normal (matching the pitch associated with each key), random (pitches selected randomly with replacement from a two-octave range), absent, or serially shifted by a lag of 1 . The serially shifted condition resulted in participants hearing pitches that matched the key pressed prior to the current keypress $(i-1)$, in synchrony with the current keypress $(i)$. Past research using serial shifts indicates that these alterations disrupt accuracy (measured by error rates) but have little effect on timing (Pfordresher, 2003a; Pfordresher \& Palmer, in press). Conversely, Finney (1997; Finney \& Palmer, 2003) and Repp (1999) both failed to find disruption when auditory feedback was absent, and Finney (1997) also found that alterations similar to the current random condition failed to disrupt production. Experiment 1 included all these conditions in the same session; it was expected that the results would replicate those found in earlier experiments.

\section{Method}

Participants. Twenty adult pianists (mean age $=23.6$, range $=15-$ 43) from the San Antonio, Texas, community participated in exchange for course credit in introductory psychology or payment. Pianists had 16.3 years of experience playing the piano (range $=8-36$ ) and 10.0 years of private piano training (range $=2.5-17$ ) on average. One pianist with few years of training (2.5) was retained because of many years of experience (34; i.e., this person was mostly self-taught); aside from this person, the minimal number of years of private training was 6 . One participant reported having absolute pitch. Eighteen participants reported being righthanded; 2 were left-handed. Fourteen participants were female, and 6 were male.

Materials. Four melodies that have been used in similar previous research served as stimulus materials (Pfordresher, 2003a; Pfordresher \& Palmer, in press). Two melodies were notated in a binary meter (2/4 time signature), and two were notated in a ternary meter ( $3 / 4$ signature). One melody for each meter condition was in the key of $\mathrm{G}$ major, and the other was in $\mathrm{C}$ major. The melodies did not contain repeating pitch patterns, so that performers would not rely on stereotyped motor movements, and none of the melodies included repeated pitches on successive events. All melodies were isochronous, comprised 12 note events, and were performed with the right hand only.

Conditions. Participants performed 2 trials for each feedback condition (normal, lag 1, random, silence) with two of the four melodies, one in a binary meter and one in a ternary meter, for 16 trials in a session. Trials were blocked first by melody and then by repetition, such that participants performed one trial of each feedback condition before continuing to its repetition. The order of feedback conditions varied randomly within each block, except that the normal feedback condition was always the first condition participants experienced after learning a new melody (i.e., at the beginning of the session and after Trial 8). The following additional factors were counterbalanced in a Latin square design that yielded four order conditions: the set of two melodies used, order of the melodies, and ordering of trials (other than the first).

Apparatus. Pianists performed melodies on a Roland RD-700 weighted-key digital piano and listened to auditory feedback over Sony MDR-7500 professional headphones at a comfortable listening level. Presentation of auditory feedback and metronome pulses, as well as MIDI data acquisition, were all implemented by the program FTAP (Finney, 2001). The piano timbre originated from Program 1 (Standard Concert Piano 1), and the metronome timbre originated from Program 126 (standard set, MIDI Key 56 = cowbell) of the RD-700.

Procedure. At the beginning of a session, participants practiced the first melody with normal feedback until they memorized it and performed without errors, after which the music notation was removed. Then participants performed at least two repetitions of that melody from memory in synchrony with the metronome, at the prescribed rate $(500 \mathrm{~ms}$ between metronome onsets). Then participants performed it with a lag-1 serial shift at a comfortable self-selected rate for another two repetitions. After this familiarization with synchronization and altered auditory feedback, participants performed at least one practice trial at the prescribed rate using the lag-1 serial shift. Then the participants completed all experimental trials for the first half of the session (eight trials in all). A brief break occurred between the two blocks, during which participants completed a questionnaire regarding musical experience. The participants then learned the second melody and performed one practice trial with lag-1 feedback before completing the second half of the session.

Each trial incorporated a synchronization-continuation paradigm (Stevens, 1886; Wing \& Kristofferson, 1973) in which altered feedback conditions occurred during the continuation phase. Participants performed each melody repeatedly throughout a trial, without pausing between repetitions. During the synchronization phase, participants performed a melody with the metronome ( $500 \mathrm{~ms}$ between metronome onsets) and normal auditory feedback. After 24 note onsets (two repetitions of the melody for error-free performances), the metronome stopped, and the participant attempted to maintain that rate during the continuation phase while one of the auditory feedback conditions took place. The continuation phase lasted for another 104 keypresses (approximately eight repetitions of the melody), after which the cessation of feedback signaled the end of the trial (except for silent feedback trials, in which the end of a trial was signaled by a high-pitched tone). Participants were instructed to adopt a legato (connected) playing style and to avoid correcting any pitch errors.

Auditory feedback conditions during the continuation phase of each trial were implemented by FTAP. During normal feedback conditions, feedback events resulted from keypresses as they would on an unaltered musical keyboard. During lag-1 trials, the FTAP program generated the pitch associated with the immediately preceding keypress. During random feedback trials, pitch events were selected at random (with replacement) from a two-octave range surrounding G5 (a pitch that falls approximately in the middle of the pitch range across all stimulus melodies). During silent feedback trials, no pitch events could be heard over headphones during the continuation phase.

Data analyses. Analyses of each trial excluded the entire synchronization phase as well as the first repetition of the melody and any events following the first produced event of the eighth repetition in the continuation phase. This resulted in six repetitions of the melody followed by a repetition of its first event (73 sequence events in performances without additions or deletions) that were analyzed for each trial. 
The primary measure of disruption was error rate (cf. Pfordresher, 2003a; Pfordresher \& Palmer, in press): the number of incorrectly produced pitch events relative to the number of produced events in the continuation phase. Errors were detected with software that compared pitches produced in performances with those that would occur in a correct performance (Large, 1993; Palmer \& van de Sande, 1993, 1995). Errors that might have resulted from learning or memory problems were filtered by the elimination of errors in the continuation phase that also occurred during the synchronization phase of the same trial. Removal of learning errors decreased error rates by less than $1 \%$ on average.

Timing of actions was measured in terms of interonset intervals (IOIs), the time elapsed between two successive keypresses. Disruption of timing was examined via variability and means of produced IOIs; variability was measured via coefficients of variation (CVs; $\mathrm{CV}=$ standard deviation of $\mathrm{IOI} /$ mean IOI within a trial). Both measures of produced timing incorporated adjustments to remove potentially spurious effects of errors and tempo drift. First, each repetition of the sequence was divided into two halves (Events 1-6 and 7-12), and, when pitch errors occurred, the error and the half sequence surrounding it were removed (cf. Pfordresher, 2003a). Following error removal, outliers were removed (defined as plus or minus three standard deviations around the mean IOI per trial), and the remaining IOIs in a trial were detrended by the addition of the mean IOI for a trial to the residual IOI values from a linear regression of IOI on sequence position (Vorberg \& Wing, 1996).

\section{Results and Discussion}

Results focus on the effect of feedback condition (normal, lag-1, random, silence) on different measures of disruption (accuracy and timing). Preliminary analyses indicated that disruption was not influenced by the melody or the musical meter performed by the participant or by order of conditions. Results for error rates, CVs (which measure timing variability), and mean IOIs (which measure production rate) are shown in Figures 2A, 2B, and 2C, respectively.

Results for accuracy, shown in Figure 2A, generally confirm the findings of previous studies that have examined these conditions separately (Finney, 1997; Pfordresher, 2003a; Repp, 1999). ${ }^{2}$ Feedback condition significantly influenced error rates, $F(3,57)=$ 8.41, MSE $=0.006, p<.01 .^{3}$ Post hoc tests (Tukey's honestly significant difference [HSD], $\alpha=.05$ ) established that the only altered auditory feedback condition producing significantly higher error rates than normal was the lag-1 condition. However, lag-1 trials did not produce higher error rates than random feedback, although they did produce higher error rates than silence. Random feedback tended to produce higher error rates than normal feedback, although this difference did not reach significance. With respect to individual participants, 15 were maximally disrupted by the lag-1 condition (compared with all other conditions), 3 were maximally disrupted by random feedback, 1 was maximally disrupted by silence, and 1 produced no errors. Thus, a significant majority of participants were maximally disrupted by lag-1 feedback in comparison with the other two altered feedback conditions, $\chi^{2}(2, N=20)=17.24, p<.01$.

Results for timing indicated more modest effects of altered feedback. Feedback condition affected CVs, $F(3,57)=4.25$, $M S E=0.007, p<.01$, but none of the pair differences was significant according to Tukey's HSD. This probably resulted from a smaller effect size for CVs than for error rates $\left(\omega^{2}=.22\right.$ for error rates, $\omega^{2}=.11$ for CVs, computed according to Keppel, 1991, p. 354). Nevertheless, the ordering of conditions with respect to $\mathrm{CV}$
A
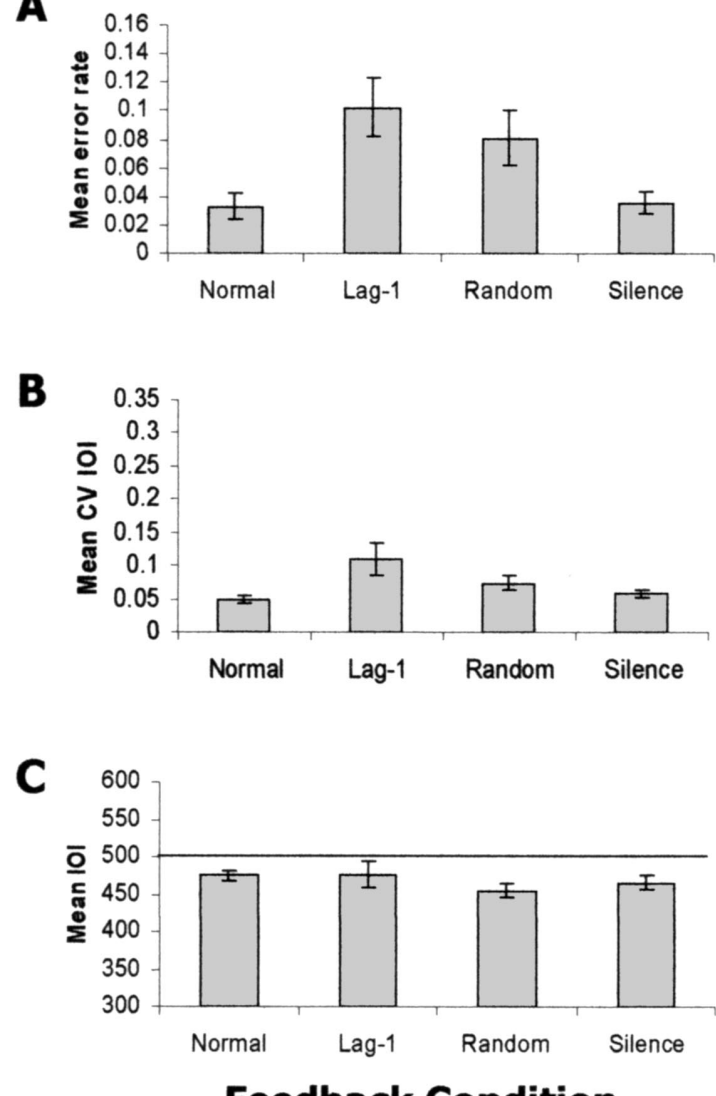

Feedback Condition

Figure 2. Disruptive effects of feedback alterations in Experiment 1 as indexed by error rates (Figure 2A), coefficients of variation (CVs; standard deviation/mean interonset interval [IOI]; Figure 2B), and mean IOIs (Figure 2C). Error bars reflect one standard error, and the horizontal bar in Figure 2C indicates prescribed tempo (500-ms IOIs).

matched that found for error rates. As found previously (Pfordresher, 2003a; Pfordresher \& Palmer, in press), mean IOI did not vary reliably as a function of feedback condition $(F<1)$.

The results of Experiment 1 converge with findings of other experiments, which confirms that their combined results reflect the influence of different feedback alterations rather than other differences across experiments. As found previously, disruptive effects were more evident in measures of accuracy than in measures of timing (Pfordresher, 2003a; Pfordresher \& Palmer, in press). Randomized feedback pitch generated slightly higher error rates than was expected, being intermediate between normal and lag 1. Still, it should be emphasized that randomized pitch did not yield error rates, on average, that could justifiably be called disruption given their nonsignificant difference from normal feedback and how few participants found random feedback more disruptive than other conditions.

\footnotetext{
${ }^{2}$ Ordinates for each plot showing results are scaled to be equivalent across experiments within each measure of disruption.

${ }^{3}$ All reported mean standard error and $p$ values reflect the GeisserGreenhouse (Keppel, 1991) correction for violations of sphericity.
} 


\section{Experiment 2}

Experiment 2 replicated the basic procedure of Experiment 1 with participants who have little or no musical training. Acquired skills can affect memory processes through repetition priming (Gupta \& Cohen, 2002) that results from correlated actionperception sequences (Bangert \& Altenmüller, 2003; Keele, Ivry, Mayr, Hazeltine, \& Heuer, 2003). Thus, the influence of auditory feedback observed in Experiment 1 might have resulted from learned associations between actions and effects acquired over long-term skill acquisition (cf. Greenwald, 1970). If so, persons without musical training might not show disruption from alterations of feedback contents. Some results from Pfordresher (2003a, Experiment 2) argue against this claim, in that persons with more musical training were less likely to be disrupted by serial shifts than were individuals with less training. However, nonpianists were not examined in that earlier experiment. The experimental procedure in Experiment 2 was modified slightly from Experiment 1 to accommodate the skill level of nonmusicians, although feedback manipulations were identical for both groups.

\section{Method}

Participants. Twenty-six adults from the San Antonio, Texas, community participated in exchange for course credit in introductory psychology (mean age $=22.0$, range $=18-34)$. Sixteen were female; 10 were male. Two participants reported having absolute pitch, and none reported hearing problems. Participants were selected at random without respect to musical experience; the only selection criteria were normal hearing and normal motor functioning, and any participants with high levels of musical training on a keyboard instrument (more than of 4 years of lessons) were excluded. Musical background was established by self-reports for years of private training and experience performing any musical instrument or singing as well as reports of any training received as a standard part of the musical curriculum (e.g., choral singing in class). Participants had 1.9 years of experience performing a musical instrument (keyboard or other) or singing (range $=0-10)$ and 0.7 years of training (range $=0-3$ ) on average. Four participants had minor levels of keyboard training, 1.3 years of training (range $=0.17-2$ ), and 2.9 years of experience $($ range $=0.5-2$ ) on average and were still considered nonpianists. Because of an experimental oversight, handedness information was not collected.

Materials. A new set of four stimulus melodies was created for Experiment 2. Each melody comprised eight events and was created so that mapping between fingers and piano keys was invariant (unlike standard music performance, in which hand position often changes). Thus, melodies included five pitches, corresponding to the white piano keys C4-G4. Two melodies began on $\mathrm{C} 5$ and initially ascended, whereas the other two began on G5 and initially descended. In addition, two of the four melodies were characterized by an alternating up-down melodic contour (fingering $=$ $1-3-2-4-5-3-4-2$ or $5-3-4-2-1-3-2-4)$, whereas the other two were created to have a smooth contour with fewer changes (fingering $=1-2$ $3-5-4-3-2-3$ or 5-4-3-1-2-3-4-3). As in Experiment 1, melodies were isochronous and were performed with the right hand.

Melodies were displayed as a row of numbers corresponding to the fingers with which the participant pressed sequential piano keys. Above each number in this row was a drawing of a hand with the to-be-moved finger highlighted (Figure 3, top panel). On the keyboard, the numbers 1-5 were arranged in a row above the corresponding piano keys, with arrows pointing from the number down to the requisite piano key (Figure 3, bottom panel).

Conditions. The conditions and design were identical to those of Experiment 1. Each participant performed two melodies that differed with respect to contour (alternating vs. smooth) and starting pitch (C5 or G5).
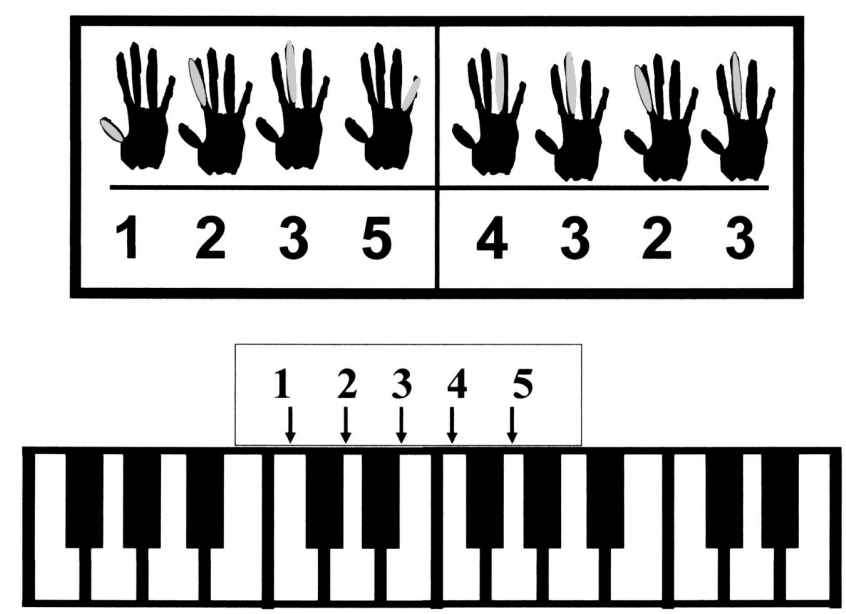

Figure 3. Example of simplified music notation used in Experiment 2 (top panel), along with the musical keyboard configuration (bottom panel).

For instance, half the participants first performed the melody that began on C5 and featured an alternating contour (Block 1) and then performed the melody that began on G5 and featured a smooth contour (Block 2).

Procedure. Instructions for performing the stimuli were adjusted to accommodate participants' general lack of musical experience. First, the experimenter explained the notation system, which was easily learned by all. Then the participants were given instructions that were designed to encourage performances similar to those of the pianists but that avoided musical terminology. Participants were instructed to keep the times between keypresses constant (i.e., rhythmical regularity) and to hold each key down until the next key was pressed (legato style). As will be seen, many nonpianists had difficulty maintaining regular timing, but all understood the instructions.

Participants also were not required to synchronize with metronome pulses at the beginning of each trial, unlike pianists in Experiment 1, because pilot studies indicated that persons without musical training find this task very difficult. Instead, participants were instructed to choose a comfortable, moderate tempo and to maintain the same speed throughout each trial. Participants heard normal auditory feedback for the first 24 keypresses of a trial (equal to 3 error-free repetitions), after which one of the experimental feedback conditions began while the participant continued to perform 12 additional repetitions, without pausing between repetitions. Nonpianists therefore produced the same number of keypresses in each trial as did pianists, although nonpianists repeated the melody more times than did pianists.

\section{Results and Discussion}

Data were analyzed as in Experiment 1. Removal of learning errors decreased error rates by less than $1 \%$ on average. Preliminary results indicated that the factors melody and repetition did not influence the disruptive effect of altered auditory feedback, so these factors are not considered further. Four participants produced more errors in the normal feedback condition than in any other condition (this included 3 persons with no musical training and 1 with some musical training). This pattern of error rates suggests that these participants had not learned the melody to an appropriate level of proficiency and/or were not sufficiently vigilant about performing optimally, and the participants were discarded from all reported analyses. Their exclusion did not qualitatively affect any of the reported findings. 
Results for accuracy, shown in Figure 4A, were similar to those for pianists: Feedback condition significantly influenced error rates, $F(3,63)=12.17, M S E=0.001, p<.01$, and post hoc tests (Tukey's HSD, $\alpha=.05$ ) indicated that lag-1 serial shifts elicited higher error rates than all other feedback conditions, which did not differ from each other. As with pianists, measures of timing showed a similar pattern but were more modest. Feedback condition had a significant effect on CVs, shown in Figure $4 \mathrm{~B}, F(3$, $63)=5.19, M S E=0.003, p<.01$. Post hoc tests revealed significantly higher CVs for lag-1 than for normal feedback, with no differences among other means. As in Experiment 1, a larger effect size was obtained for error rates $\left(\omega^{2}=.29\right)$ than for CVs $\left(\omega^{2}=.13\right)$. The effect of feedback on mean IOIs fell short of significance $(p=.06)$.

To address the issue of musical experience more thoroughly, I directly compared the results of Experiment 2 with those from Experiment 1. The participants of Experiment 2, who reflect a more heterogeneous population than those in Experiment 1, were divided into two groups for these analyses. One group included participants who had some experience playing a musical instrument or singing $(n=8)$, and the other group included participants who had no musical experience, even in the general curriculum
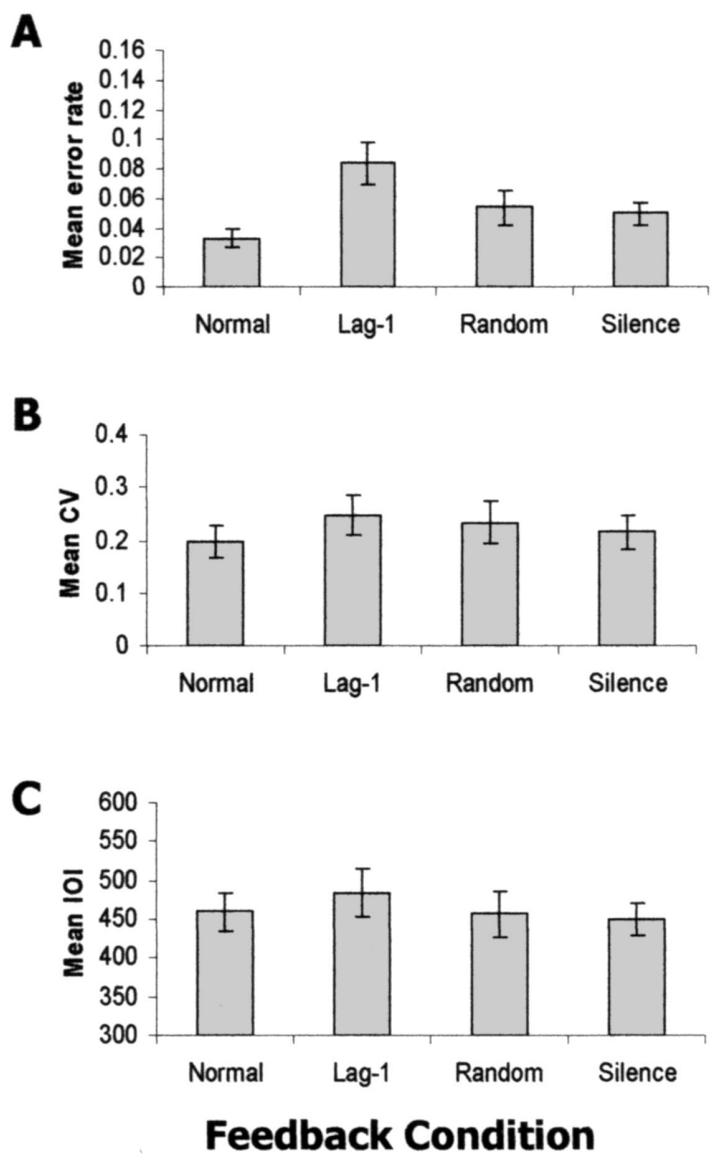

Figure 4. Disruptive effects of feedback alterations in Experiment 2 as indexed by error rates (Figure 4A), coefficients of variation (CVs; standard deviation/mean interonset interval [IOI]; Figure 4B), and mean IOIs (Figure 4C). Error bars reflect one standard error.
( $n=10)$. Four participants who had some experience in the general curriculum but no experience with a specific instrument or voice were excluded from these analyses.

Each measure of disruption was analyzed as a function of the three groups described previously (pianists, nonpianists with minimal experience, nonpianists with no experience) and feedback condition, a $3 \times 4$ mixed factorial. None of the analyses detected a Group $\times$ Feedback Condition interaction, which indicates that the effects of auditory feedback conditions on different measures of disruption were not influenced by musical experience. The only main effect for group was found for CVs, $F(3,35)=7.14, M S E=$ $0.053, p<.01$. Post hoc tests (Tukey's HSD, $p<.05$ ) indicated that participants in Experiment 1 were significantly less variable $(M=0.072)$ than participants with no training from Experiment 2 $(M=0.239)$.

Because participants in Experiment 2 were allowed to choose their own tempo, it is possible that tempo (rate of production) influenced patterns of errors. However, several additional analyses suggested that performance tempo did not influence results. First, participants were separated into quartiles on the basis of their mean tempo. Mean error rates revealed the same pattern of results within each quartile. Second, speed-accuracy analyses were conducted for each participant through correlations between mean IOI and error rates for each trial. Only 2 participants showed a significant negative relation. Furthermore, speed-accuracy analyses conducted across subjects and trials for each feedback condition failed to yield a significant negative relation within any feedback condition.

The results of Experiment 2, like those of Experiment 1, demonstrate that disruption from altered feedback pitches was most pronounced when the feedback sequence was structurally identical to the planned sequence but was serially displaced. The fact that this was found for individuals across a wide range of musical training suggests that this kind of perception-action coordination does not result from extensive training at the piano and can be exhibited within a brief training period.

\section{Experiment 3}

Experiment 3 explored the prediction that disruption increases as the sequence of altered feedback events becomes increasingly similar in structure to the planned sequence, as described in the introduction. Lag-1 serial shifts and randomized feedback can be considered as two ends of a continuum of decreasing similarity between a planned sequence and a sequence of altered events. Lag-1 serial shifts maintain the planned set of pitch events and their serial order but differ from the planned sequence with respect to the sequential alignment of planned versus feedback events. Random feedback sequences, by definition, are independent of the planned sequence. Moreover, randomized pitch sequences do not present the performer with a coherent melodic structure and may easily be dissociated from actions by virtue of their formless quality. ${ }^{4}$ Experiment 3 explores this implication by including altered feedback sequences of intermediate similarity between lag-1 and random feedback conditions.

Experiment 3 was also designed to address alternative explanations for the difference found between lag-1 and randomized

\footnotetext{
${ }^{4}$ I thank Bruno Repp for pointing out this possible account.
} 
feedback in Experiments 1 and 2. First, the range of perceived pitches was wider in the random feedback condition (two octaves) than in the produced melodies (just over one octave for pianists and seven semitones for nonpianists). It is possible that the wider pitch range resulted in an experience of the feedback sequence as separate from the action sequence, which led to reduced disruption. For instance, Gates and Bradshaw (1974) reported disruption of piano performance when performers concurrently heard a recorded performance that was in the same pitch range as the performed piece. Second, it is possible that interference results whenever one hears a repeating pattern that differs from the produced pattern, whereas randomized feedback is unlikely to result in a regular repeating pattern.

These issues were addressed by two new conditions in Experiment 3, which were included along with the normal, lag-1, and randomized feedback conditions. I formed one new condition, called random-same, by scrambling the order of pitches in the planned sequence such that the resulting feedback sequence did not match the higher order structure of the planned sequence. If disruption scales with similarity, the random-same condition should disrupt production, but to a lesser degree than lag-1 serial shifts. I produced the second new condition, called randomdifferent, in the same way as random-same, except that I shifted every component pitch up or down one semitone so that no single feedback pitch matched any of the produced pitches in the melody. Feedback melodies therefore did not match the tonal implications presented in planned melodies (cf. Krumhansl, 1990, 2000). These new conditions were therefore not truly random but were assumed to have a randomlike quality by virtue of their dissimilarity to planned sequences. If auditory feedback merely results from hearing some repeating sequence other than the planned one or merely reflects the pitch range present in the altered feedback sequence, all conditions except for the original randomized condition should prove equally disruptive.

\section{Method}

Participants. Fourteen adult pianists (mean age $=26.4$, range $=$ $18-42$ ) from the San Antonio, Texas, community participated in exchange for payment. Of these, 4 had also participated in Experiment 1. Participants had 9.9 years of private piano training (range $=4-16$ ) and 17.7 years of experience playing the piano (range $=6-35$ ) on average. All participants reported being right-handed. Eight participants were female, and 6 were male.

Materials and conditions. Participants experienced five auditory feedback conditions, which used the same four melodies that were used in Experiment 1. Three conditions were identical to those used in Experiments 1 and 2: normal, lag-1 serial shifts, and random. Two additional auditory feedback conditions were created in the following way for each melody. I created random-same conditions from randomly permuted orderings of pitches in the stimulus melody. The feedback sequence thus comprised the same individual pitches as the planned melody, but in a different order that was maintained across repeated performances of the melody. I created random-different feedback conditions from a new permutation of the melody by randomly shifting each individual pitch up or down one semitone so that none of the auditory feedback pitches matched any of the produced pitches across the melody. I compared each permuted melody with the original to ensure that a predictable perception-action relation did not occur by chance. Figure 5 shows an example of how random-same and random-different conditions were generated. In this

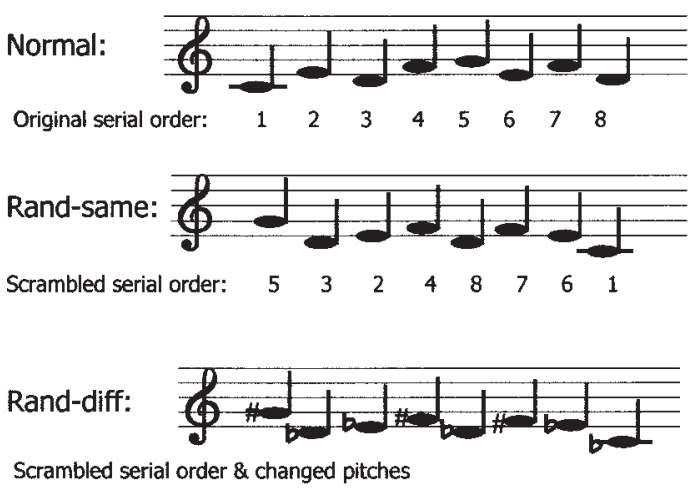

Figure 5. Example of changes to the performed melody in random-same (Rand-same) and random-different (Rand-diff) conditions in Experiment 3.

example, the same permuted order is used for both altered feedback conditions, whereas in practice, two different permutations would be used.

Feedback sequences for random-same and random-different conditions were thus designed to resemble the planned sequence in certain statistical ways but to differ from planned sequences with respect to serial structure (the ordering of events). I verified the fact that feedback sequences were dissimilar to planned sequences with respect to serial structure by computing correlations between planned and feedback sequences across serial position. I calculated different correlations using MIDI pitch numbers (which code pitch height in semitones) and melodic transitions (event-toevent pitch differences in semitones). These correlations were nonsignificant, and their average was near zero. Conversely, random-same sequences featured the same set of pitches as those that participants planned to perform, and random-different sequences featured a set that was similarly constrained with respect to the range of possible pitches and the number of different pitch classes included. Furthermore, visual inspection of distributions for melodic intervals (difference in semitones between successive pitches) across feedback conditions indicated that highly similar sets of melodic intervals were included in all feedback conditions except for the true random condition, again irrespective of serial position.

Procedure. The procedure for individual trials was identical to that used in Experiment 1, except that pianists were given instructions to perform at a slow moderate tempo and no metronome pulses were given during the synchronization phase (which refers to the first 24 keypresses here). This was done to better equate the procedures for pianists and nonpianists.

During the continuation phase of each trials, feedback events for the normal, lag-1, and random conditions were selected as in Experiments 1 and 2. The random-same and random-different conditions were implemented such that each keystroke triggered the feedback pitch at the associated sequence position.

\section{Results and Discussion}

Data were analyzed as in Experiments 1 and 2. Figure 6A shows mean error rates across feedback conditions for Experiment 3. Conditions are ordered according to similarity between produced and auditory feedback sequences, with maximal similarity occurring for normal feedback and minimal similarity occurring for random feedback. As in the earlier experiments, no differences emerged as a function of the notated musical meter. A single-factor analysis of variance (ANOVA) on error rates yielded a main effect of feedback condition, $F(4,52)=8.43, M S E=0.003, p<.01$. Post hoc tests (Tukey's HSD, $\alpha=.05$ ) indicated that the lag- 1 and random-same conditions increased error rates relative to the nor- 
A

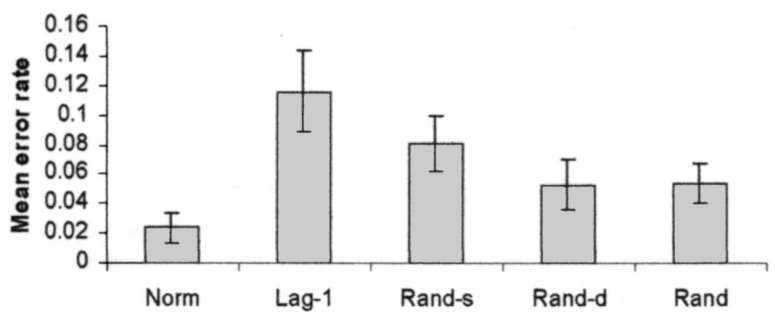

B

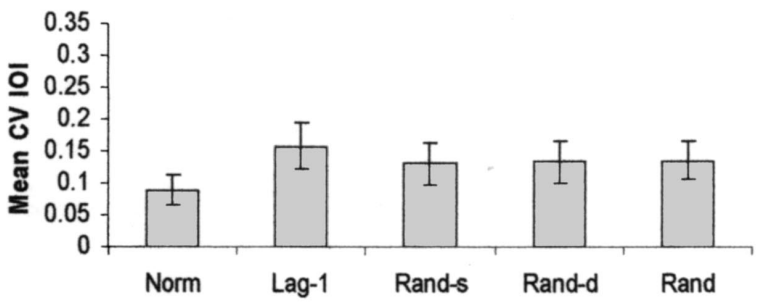

C

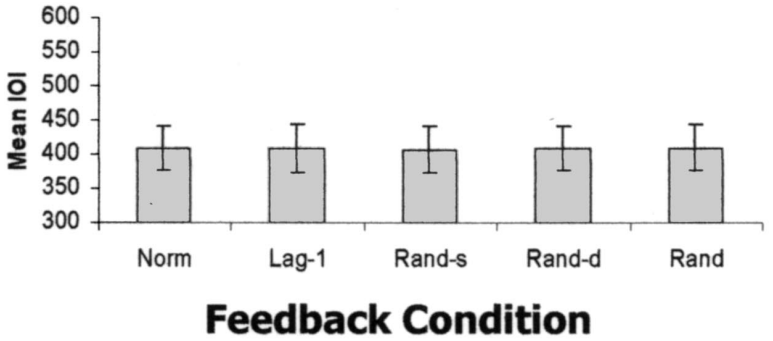

Figure 6. Disruptive effects of feedback alterations in Experiment 3 as indexed by error rates (Figure 6A), coefficients of variation (CVs; standard deviation/mean interonset interval [IOI]; Figure 6B), and mean IOIs (Figure 6C). Error bars reflect one standard error. Norm = normal; Rand-s $=$ random-same; Rand-d = random-different; Rand = random.

mal feedback condition and did not differ from each other. Furthermore, error rates for the lag-1 condition significantly exceeded those for the random-different and random conditions, but error rates for the random-same condition did not. The results of these post hoc tests thus suggest that disruption of accuracy was maximal for the lag-1 condition, intermediate for the random-same condition, and negligible for the random-different and random conditions. This implication was confirmed by a linear trend across altered feedback conditions ordered according to their structural similarity, as in Figure 6A, $F(1,13)=9.72, M S E=0.003, p<$ .01 ; the quadratic trend was not significant.

Analyses of produced timing are shown in Figures 6B and 6C. The influence of auditory feedback on CVs fell short of significance $(p=.06)$, although the highest CVs were again found for the lag-1 condition. Feedback manipulations did not influence mean IOIs $(F<1)$.

Additional analyses tested whether performance tempo influenced disruption of accuracy, as in Experiment 2. Performers were separated into quartiles on the basis of mean overall tempo ( $n=3$ for the two extreme quartiles, $n=4$ for the inner two quartiles). Mean error rates for each quartile followed the basic pattern shown in Figure 6A. One minor exception is that mean error rates for random feedback in the second fastest quartile were similar to those for the random-same condition and were higher than those for the random-different condition. Speed-accuracy analyses conducted on individual participants revealed no significant negative relations. Speed-accuracy analyses conducted across subjects and trials for each feedback condition failed to yield a significant negative relation for any feedback condition except lag 1 , which did yield a significant negative relation $(r=-.37, p<.01)$. Overall, the results do not seem to be confounded by participants' choice of performance tempo.

The results of Experiment 3 suggest that disruption from altered feedback scales with the similarity between produced and perceived sequences. During normal feedback, associations between planned actions and feedback reinforce each other. However, similarity proves a liability when feedback is altered, primarily when the feedback sequence is structurally identical to but serially displaced from the planned sequence (the lag-1 condition). The apparent absence of disruption from the random-different condition indicates that performers are sensitive to matches between the planned and perceived events, not just to the overall range of pitches in auditory feedback sequences. Again, the strongest influence of auditory feedback was seen in error rates, which adds further support for the idea that alterations of pitch information disrupt performance more than the timing of actions.

\section{Experiment 4}

Experiment 4 adopted the basic methodology of Experiment 3 but included nonpianists, as did Experiment 2. Random-same and random-different conditions were derived from the simplified melodies used for nonpianists. On the basis of the similar results found for both groups across Experiments 1 and 2, it was predicted that the results of Experiment 4 would converge with those from Experiment 3 .

\section{Method}

Participants. Twenty-three adults (mean age $=19.2$, range $=17-26$ ) from the San Antonio, Texas, community participated in exchange for course credit in introductory psychology. None of the participants had participated in the preceding experiments. Fourteen participants were female, and 9 were male. Handedness information was obtained from 15 participants, all of whom reported being right-handed. Participants had 4.1 years of experience performing an instrument or singing (range $=0-19$ ) and 2.1 years of training (range $=0-9$ ) on average. Four participants had minor levels of keyboard training, 2.3 years of training (range $=1-4$ ), and 5.0 years of experience (range $=2-8$ ) on average but were still considered nonpianists.

Materials and conditions. The materials were identical to those used in Experiment 2. The conditions matched those in Experiment 3, except that the random-same and random-different conditions were based on the melodies used by nonmusicians. As in Experiment 2, these new conditions produced feedback conditions that were uncorrelated with planned melodies in terms of individual pitches or transitions between pitches (mean $r=$ .03), although statistical properties of random-same and random-different conditions that do not reflect sequential relations (distribution of pitch classes and pitch intervals) were similar to planned sequences.

\section{Results and Discussion}

Data were analyzed as in other experiments. The data of 3 participants were discarded, as was done in Experiment 2, because 
these participants made the most errors in the normal feedback condition; their exclusion did not influence significance levels of results.

Figure 7A shows error rates across the five feedback conditions in Experiment 4. Feedback condition significantly influenced error rates, $F(4,76)=8.99, M S E=0.001, p<.01$. Post hoc tests (Tukey's HSD, $\alpha=.05$ ) supported a pattern of results like that found for pianists: Only lag-1 serial shifts significantly increased error rates relative to normal feedback, and serial shifts elicited higher error rates than other altered feedback conditions, except random-same. As with pianists, mean error rates decreased with increasing dissimilarity between produced and altered feedback conditions, which led to a significant linear trend across altered feedback conditions, $F(1,19)=16.21, M S E=0.001, p<.01$; the quadratic trend was nonsignificant.

CVs (Figure 6B) varied as a function of feedback condition, $F(4,76)=5.61, M S E=0.006, p<.01$. Post hoc tests (Tukey's HSD, $\alpha=.05)$ indicated that every altered feedback condition elevated CV above normal feedback except for the randomdifferent condition. Effect size was again higher for error rates $\left(\omega^{2}=.24\right)$ than for CVs $\left(\omega^{2}=.16\right)$. Feedback condition did not

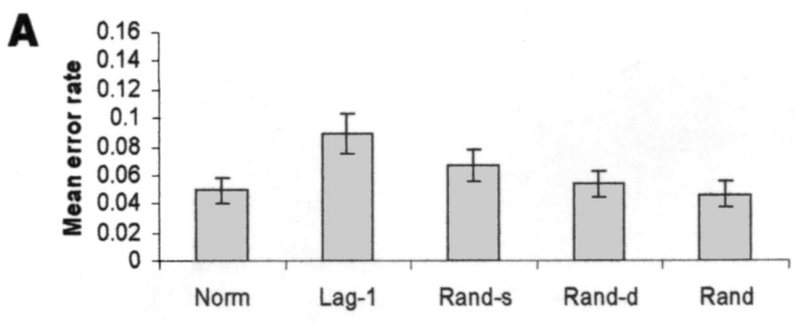

B
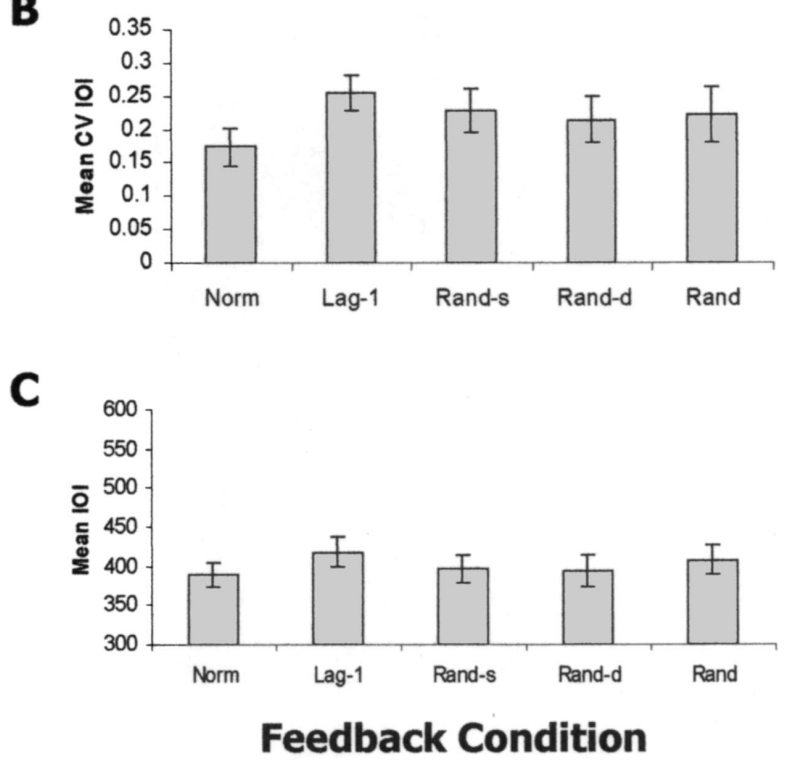

Figure 7. Disruptive effects of feedback alterations in Experiment 4 as indexed by error rates (Figure 7A), coefficients of variation (CVs; standard deviation/mean interonset interval [IOI]; Figure 7B), and mean IOIs (Figure 7C). Error bars reflect one standard error. Norm = normal; Rand-s = random-same; Rand-d = random-different; Rand $=$ random. significantly affect mean IOI $(F<1)$. Note that the mean IOI was similar to that of the pianists in Experiment 3.

The role of musical experience in the combined results of Experiments 3 and 4 was addressed as in the results of Experiments 2 and 3. Participants in Experiment 4 were divided into two groups: those with some experience on an instrument or voice ( $n=$ 13 ) and those with no experience $(n=6)$. One participant who had some experience in the general curriculum but no experience with a specific instrument or voice was excluded. As before, variability in each measure of disruption was analyzed as a function of these three groups and feedback condition. None of the analyses yielded a Group $\times$ Feedback Condition interaction, which confirms earlier results. In addition, group did not yield any main effects. Although individuals without musical training generated more variable timing (higher $\mathrm{CVs}$ ) than did pianists, the main effect of group on $\mathrm{CVs}$ fell short of significance $(p=.10)$.

A series of analyses was run to test whether performance tempo influenced disruption of accuracy. As in Experiments 2 and 3, no influence of performance tempo on patterns of error rates emerged, and neither did speed-accuracy trade-offs for individual participants or within feedback conditions.

The results of Experiment 4 largely replicate those of Experiment 3 , thereby adding further support for the idea that disruption from altered feedback does not result from the disruption of action-effect associations that are acquired during prolonged musical training. Furthermore, disruption continued to be most clearly reflected in error rates, which generated the largest effect size (compared with $\mathrm{CVs}$ ) and most clearly reflected perception-action similarity.

\section{Experiment 5}

Experiments 1-4 document similar responses to altered feedback for pianists and nonpianists. The proposed theoretical framework suggests that these similarities are found because the shared representation generates sensitivity to correlations between planned and perceived sequences across domains. An alternative account relies on learned associations between actions and perceived consequences. Although nonpianists could not have acquired such associations over long-term training, it is possible that they formed short-term associations at the beginning of the session in Experiments 1-4 while learning the melody with sound. Recent electroencephalographic data indicate that persons without piano training can acquire movement-perception associations in a very short time frame, provided that these relations are consistent (Bangert \& Altenmüller, 2003). Experiment 5 addresses this issue with nonpianists by having participants learn the musical sequence without sound.

A related issue has to do with the potentially disruptive influence of the transition from normal to altered auditory feedback that occurred at the beginning of each trial (i.e., the transition from synchronization to continuation). Perhaps the greater disruption found for the randomsame condition than for the random-different condition happened because the transition was more subtle for the former than for the latter, which would not have allowed performers to apply a strategy such as ignoring the auditory feedback events (which many participants reported using) as quickly as would be optimal. Experiment 5 addresses this issue by presenting the experimental auditory feedback condition throughout the entire trial rather than having all trials begin with normal feedback. 


\section{Method}

Participants. Twenty-five adults (mean age $=20.3$, range $=18-43$ ) from the San Antonio, Texas, community participated in exchange for course credit in introductory psychology. None had participated in the preceding experiments. Twenty participants were female, and 5 were male. Twenty participants reported being right-handed, and 5 were left-handed. Three participants reported having absolute pitch. Participants had 4.7 years of experience performing an instrument or by voice (range $=0-25$ ) and 1.7 years of training (range $=0-6$ ) on average. Two participants reported minimal years of training on the piano $(M=2.0)$ but were still considered nonpianists.

Procedure. Two aspects of the procedure of Experiment 5 differed from that of Experiment 4. First, participants learned each melody without sound; the experimenter gave the participant the same instructions otherwise. Following practice, participants performed the melody without notation or sound, and the experimenter watched their finger movements to ensure that the piece was learned and performed correctly (finger movements were also observed for the first two repetitions). Second, the experimental feedback condition began at the beginning of each trial and continued until the end of the trial; there was no transition from normal to altered feedback within a trial. Overall trial lengths were the same as in earlier experiments.

\section{Results and Discussion}

Data were processed exactly as in Experiment 4 despite the absence of a synchronization phase; the data of 3 participants were discarded because these participants made the most errors in the normal feedback condition, and their exclusion did not influence results. Figure 8 shows patterns of error rates from Experiment 5, along with error rates from Experiment 4 (also shown in Figure 7) for purposes of comparison.

Auditory feedback conditions yielded similar effects on error rates as in Experiment 4, with the exception that slightly higher error rates were elicited by random feedback in Experiment 5. Feedback condition significantly influenced error rates, $F(4,84)=$ 4.33, MSE $<0.001, p<.05$. Post hoc comparisons (Tukey's HSD, $\alpha=.05)$ revealed that lag-1 serial shifts generated significantly higher error rates than the normal or random-different conditions but did not differ from the random-same condition. Unlike in Experiment 4, lag-1 serial shifts did not generate higher error rates than random sequences, although the linear trend across altered

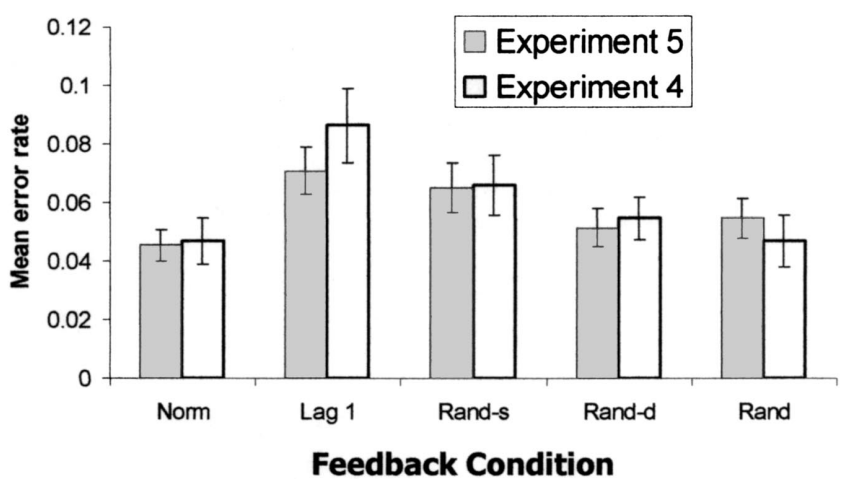

Figure 8. Error rates for Experiments 4 and 5. Error bars reflect one standard error. Norm $=$ normal; Rand-s $=$ random-same; Rand-d $=$ random-different; Rand $=$ random. feedback conditions was significant, $F(1,21)=4.93, M S E=$ $0.001, p<.05$, and the quadratic trend was not significant. An ANOVA on CVs showed that timing variability also varied with feedback condition, $F(4,84)=8.99, M S E=0.002, p<.01$. Post hoc tests determined that all altered conditions yielded higher $\mathrm{CVs}$ than did normal feedback. As in other experiments, no influence on mean IOIs was found.

Next, we compared error rates (our primary measure of disruption) across Experiments 4 and 5. An ANOVA that compared the error data of Experiments 4 and 5 (a $2 \times 5$ mixed model) failed to yield a main effect of experiment, and the Experiment $\times$ Feedback Condition interaction fell short of significance $(p=.08)$. Similarly, pair contrasts across experiments for mean error rates within each feedback condition yielded no significant results. One subtle difference across experiments is that error rates for lag- 1 serial shifts were $2 \%$ higher on average for Experiment 4, when a transition was present, than for Experiment 5. Differences in error rates across experiments for other altered feedback conditions were less than $1 \%$ on average. Thus, although random feedback generated slightly higher errors, relative to other conditions, in Experiment 5 than in Experiment 4, this difference was not reliable in cross-experiment comparisons.

The results of Experiment 5 indicate, overall, that commonalities observed across pianists and nonpianists did not result either from exposure to sound during learning or from transitions between normal and altered phases of a trial. In contrast to the present findings, which show similar performance regardless of whether participants were exposed to sound at the beginning of the session, Finney and Palmer (2003) found that exposure to sound during encoding facilitated retrieval of melodies. Thus, the current results suggest that disruption can occur even without the facilitating influence of sound during learning. Moreover, although altered feedback pitch may well disrupt retrieval (cf. Pfordresher \& Palmer, in press), this disruption does not occur because of associations gained during learning of the melody.

\section{Experiment 6}

A final experiment (suggested by Bruno Repp, personal communication, March 21, 2005) was undertaken to further address whether nonpianists relate actions to perceived pitches on a musical keyboard. Pianists in Experiment 6 learned a single melody without sound (as in Experiment 5) and then performed the melody under different feedback conditions, which were the same as those included in Experiments 3-5. After each trial, the performer rated the similarity of the perceived melody to the kind of melody that he or she would expect to result from the planned sequence of keypresses.

\section{Method}

Participants. Eight adults (mean age $=19.6$, range $=18-26$ ) from the San Antonio, Texas, community participated in exchange for course credit in introductory psychology. None of the participants had participated in the preceding experiments. Three participants were female, and 5 were male. Seven participants reported being right-handed, and 1 was left-handed. No participants reported having absolute pitch. Participants had 6.0 years of experience performing an instrument or by voice (range $=0-17$ ) and 3.1 years of training (range $=0-8$ ) on average. Most important, all but 1 participant reported no experience playing the piano, and the remaining participant reported only 1 year of experience and no years of formal training. 
Procedure. As in Experiment 5, participants practiced the stimulus melody without sound, and each feedback condition was present for the entirety of a trial. During practice, participants were told to focus on finger movements. Participants learned and performed only one melody in Experiment 6 to reduce the possibility of memory confusions in the similarity rating task. During lag-1 and normal feedback trials, each feedback pitch presented the planned (i.e., correct) event for that sequence position (as in the random-same and random-different conditions); this change was implemented so that participants would rate the similarity of sounds to their planned rather than their produced performance.

An additional rating task was used at the end of each trial. After a trial was completed, a high-pitched bell sounded. Following this cue, the participant pressed a single key on the keyboard to rate the similarity of the perceived melody to "the kind of melody you might expect to hear when performing the melody on a normal piano." The rightmost key on the keyboard was labeled "same," the leftmost key was labeled "different," and intermediate keys were used to represent intermediate levels of similarity. Participants were instructed to respond on the basis of their instincts, even if they had no piano experience, and to use extreme ratings. No sound resulted from the keypress used to rate similarity.

\section{Results and Discussion}

Analyses in Experiment 6 focused on rating responses. Responses were coded by MIDI key number (ranging from 36 to 88) and transformed to a 100-point scale; mean ratings are shown in Figure 9. Ratings of similarity varied significantly across feedback conditions, $F(4,28)=14.47, M S E=0.062, p<.01$. Post hoc tests (Tukey's HSD, $\alpha=.05$ ) verified that normal and lag-1 feedback did not differ from each other but differed from all other feedback conditions, which likewise did not differ from each other. There was a significant linear trend across all conditions, $F(1,7)=$ $26.89, M S E=0.062, p<.01$; no other trends were significant.

Experiment 6 adds further support to the idea that nonpianists are sensitive to perception-action relations. Furthermore, it is significant that normal and lag-1 feedback conditions, which differed strikingly with respect to disruption in Experiments 1-5, yielded highly comparable similarity ratings in Experiment 6 . Moreover, the rank order of similarity relations across altered feedback sequences matched the rank order of error rates in Experiments 3-5.

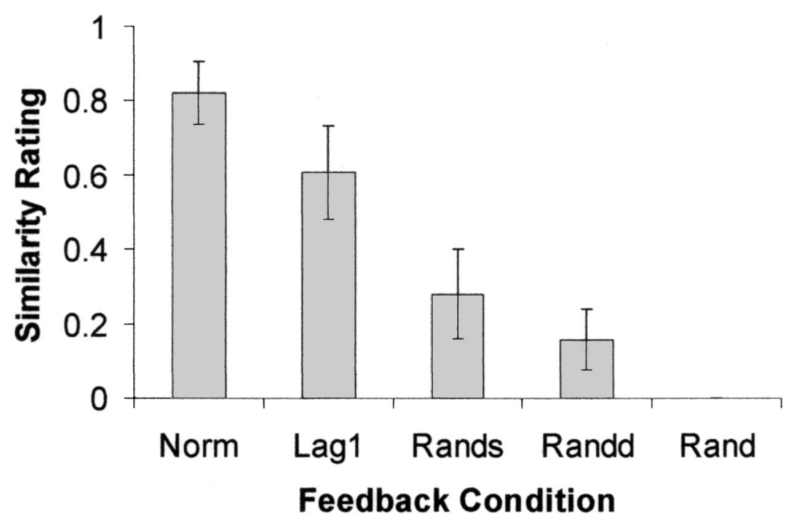

Figure 9. Similarity ratings for feedback conditions in Experiment 6. Error bars reflect one standard error. Norm $=$ normal; Rands $=$ randomsame; Randd $=$ random-different; Rand $=$ random.

\section{General Discussion}

Results converge on two conclusions. First, music performance can be disrupted by altered feedback pitch contents, but disruption is contingent on structural similarity between the planned sequence and the sequence formed by altered auditory feedback events. Although optimal performance occurs when the planned and perceived sequences match and are sequentially coincident (normal auditory feedback), the same kind of similarity disrupts performance when the serial positions of planned and perceived events are shifted relative to each other (serial shifts). However, performance was unhindered by the absence of feedback and was not significantly disrupted by randomized pitches. Intermediate disruption emerged when feedback alterations presented a scrambled version of the planned sequence, one in which the constituent pitches matched produced pitches but did not adhere to the same kind of higher order structure formed by the ordering of pitch events. Other research has established the disruptive effects of serial shifts (Pfordresher, 2003a; Pfordresher \& Palmer, in press) as well as absence of disruption from random feedback or silence (Finney, 1997; Repp, 1999). However, the present study is the first to demonstrate this dissociation within participants, and, more important, to establish the influence of overall structural similarity between the planned sequence and the sequence of auditory feedback events.

The second main conclusion is that formal musical training does not influence which alterations of feedback contents disrupt performance. The only measure on which pianists and nonpianists differed was timing variability $(\mathrm{CV})$, but the skill level of different groups never interacted with feedback condition. Thus, pianists and nonpianists differ with respect to temporal control of movements, but this control is separate from the kind of perceptionaction coordination that serial shifts disrupt (cf. Pfordresher, 2003a). The fact that nonpianists were disrupted by conditions similar to those that disrupt pianists suggests that the kind of action-effect coordination with which altered feedback interferes constitutes a general characteristic of how the mind represents the structure of produced or perceived sequences. Moreover, these results suggest that action-effect associations may be more intrinsic to the system than is implied by earlier associationist accounts (see Greenwald, 1970; Lashley, 1951, for further discussion).

\section{The Coordination of Perception and Action}

The present study continues a recent line of research that has addressed the role of auditory feedback contents, in particular pitch information during music performance (e.g., Finney, 1997; Pfordresher, 2003a; Pfordresher \& Palmer, in press). The current paradigm departs from much previous work in that feedback events are always presented in synchrony with produced events. As demonstrated in the current experiments, changes of pitch information can disrupt performance, although important qualifications exist concerning the conditions under which this disruption occurs.

The mere fact that changes of pitch disrupt performance discredits an alternative view-that auditory feedback does not influence the planning of sequence production but only influences motor execution (e.g., Howell et al., 1983). The present results fit better with an explanation that focuses on matches between 
planned and perceived outcomes of actions. This proposal is supported by the fact that alterations of feedback contents primarily influence the performer's ability to select the correct responses (i.e., they increase pitch error rates) but do not have such strong effects on the timing of movements.

The degree to which certain alterations cause more or less disruption has implications for the nature of perception-action coordination, as suggested earlier. In this context, it is worth noting that experimental factors influencing the degree of disruption differ for feedback manipulations that influence feedback synchrony, such as DAF, and alterations of feedback contents in synchronous feedback, such as those manipulated here. For manipulations like DAF, the primary variable determining disruption is the amount of asynchrony, with disruption increasing up to a particular magnitude and decreasing thereafter (e.g., Fairbanks \& Guttman, 1958; Finney \& Warren, 2002; Howell et al., 1983). Such effects have been interpreted in light of temporally based execution processes, possibly regulated by the cerebellum (Howell, 2001). By contrast, research that has varied the amount of serial shift (i.e., number of events separating the current event and the event associated with auditory feedback) has not shown differences across the degree of serial separation between the planned event and the position associated with auditory feedback for distances up to three events in the past or future (Pfordresher, 2003a; Pfordresher \& Palmer, in press). Instead, the primary variable determining disruption from altered pitch appears to be the higher order organization of the sequence of feedback pitches relative to the planned sequence, as demonstrated by the current data. Thus, serial shifts appear to disrupt a qualitatively different aspect of perception-action coordination than do manipulations that cause asynchronies between actions and feedback (Pfordresher, 2003a). It is plausible that serial shifts disrupt memory-based processes that are related to planning rather than execution-based processes.

The current results are consistent with the idea that perception and action share a common representation (e.g., Hommel et al., 2001; MacKay, 1987). In this account, disruption results when perceived note events match events other than the current event. Auditory feedback in these circumstances adds activation for an event that is supposed to be less active than the event associated with the action that was just produced, resulting in interference between the activations of the current event and the event that auditory feedback matches. In the case of random feedback or the random-different feedback condition, perceived events rarely (if ever) match events intended for other serial positions. In the case of silence, a performer can still plan the sequence on the basis of activations of events planned for production (i.e., this is not a "feedback control" explanation).

The fact that less disruption resulted from the random-same feedback condition than from lag-1 serial shifts suggests that performers are sensitive to perception-action relations with respect to both individual events and the serial structure of events across the sequence (see Figure 1). Thus, lag-1 serial shifts may add activation both to individual events and to transitions between events (designated by serial order) intended for other positions. By contrast, random-same feedback alterations would add activation only to individual events and cause less interference. Past research indicates that event transitions, such as melodic contour (the patterns of upward and downward pitch motion), are particularly salient with respect to the perception of musical structure (e.g.,
Boltz \& Jones, 1986; Dowling, 1978; Jones, 1987; Jones \& Pfordresher, 1997; Pfordresher, 2003b). Another implication of this interpretation is that representations underlying sequence production and sequence perception may incorporate the distinction between item and order memory (e.g., Bjork \& Healy, 1974; Healy, 1974).

One possible alternative explanation for the difference between lag-1 and random-same feedback alterations focuses on the average separation between the planned event and the position associated with altered feedback events, which was always one event for the lag-1 conditions but varied for random-same conditions. However, this explanation is unlikely to account for the present results. Feedback events in random-same patterns were separated from the current event by nearly one event on average, just like the serial shifts, for the melodies performed by nonmusicians (mean absolute distance $=1.1$ ). The mean separation between events was significantly greater than one for the random-same patterns performed by pianists $(M=2.3), t(48)=2.55, p<.05$. However, past research has demonstrated similar disruption from serial shifts stemming from past or future events within a distance of three, as discussed earlier (Pfordresher, 2003a; Pfordresher \& Palmer, in press).

The link between perception and performance of music proposed here converges with results of other research in music cognition and human performance. For instance, listeners often demonstrate expectations for temporal fluctuations in music that resemble the same fluctuations that performers add (e.g., Repp, 1995, 1998a, 1998b). These results suggest that performers and listeners incorporate a similar representation of the relation between timing and musical structure. Similarly, research on choice reaction time in nonmusical behaviors indicates facilitation of performance from compatible and interference from incompatible stimulus-response configurations (see Cho \& Proctor, 2003; Hommel \& Prinz, 1997, for reviews) and response-effect configurations (Elsner \& Hommel, 2001; Keller \& Koch, 2005; Kunde 2001; Kunde, Koch, \& Hoffmann, 2004; Stöcker et al., 2003).

\section{The Role of Musical Skill}

The second main issue addressed by the current research concerns the role of musical skill in mediating perception-action relations during music performance. The current results show clear similarities across participants regardless of musical training. These results are particularly striking given that (a) the musical behavior was keyboard performance rather than one in which nonmusicians commonly engage, like singing, and (b) the present manipulations of auditory feedback probed abstract levels of representation, such as the serial order of events (Lashley, 1951), rather than simple matches between planned and perceived events. Furthermore, the results of Experiment 5 suggest that actioneffect associations formed during learning do not account for these results.

The reported similarities across pianists and nonpianists suggest that the sequential structure of music, as performed or perceived, is served by cognitive mechanisms that are shared with other domains. Music, language, gesture, and other serial behaviors organize motor gestures within a higher order structure that may be characterized by a generative grammar (e.g., Chomsky, 1957; Lerdahl \& Jackendoff, 1983). It is possible that all these behaviors 
reflect domain-general cognitive mechanisms that organize complex event sequences (e.g., Jones, 1976, 1990; Levitin \& Menon, 2003; Pinker, 1997; Wallin, Merker, \& Brown, 2001; Zatorre \& Peretz, 2001). By this account, it should not be surprising that nonpianists are disrupted by altered feedback sequences that share structural characteristics with the planned sequence, because the cognitive representation they use to perceive and plan that musical sequence is fundamentally the same as the representation used to organize other action sequences, such as speech.

Taken together, the current results suggest that the acquisition of music performance skills does not substantially influence how a person integrates auditory feedback into a plan of action, despite the many changes that musical skill causes. In this context, it is noteworthy that the most substantial difference between trained and untrained performers was in the overall difference in timing variability, which reflects the temporal precision in motor control that is acquired through musical training (Drake \& Palmer, 2000; cf. Ericsson \& Charness, 1994). However, as mentioned earlier, this difference between groups does not appear to modulate disruption from feedback manipulations.

In contrast to the current similarities between pianists and nonpianists, other studies have documented substantial changes due to training in music performance in neural functioning (Bangert \& Altenmüller, 2003; Haslinger et al., 2004), anatomy (e.g., Schlaug, Jäncke, Huang, \& Steinmetz, 1995), cognitive planning (Drake \& Palmer, 2000; Palmer \& Drake, 1997), and timing (Drake, Jones, \& Baruch, 2000; Drake, Penel, \& Bigand, 2000). In particular, some recent studies have documented differences across musicians and nonmusicians with respect to the way auditory feedback is processed (Bangert \& Altenmüller, 2003; Keller \& Koch, 2005). Given the similarities across groups in the current data, why have other studies revealed striking differences across groups? I suggest that these differences can be accommodated by the idea that musical skill fine tunes connections within a domain-general representation. Whereas the designs used here identify conditions under which the system breaks down, other studies have focused on the efficiency with which the system operates. For instance, Keller and Koch (2005) found evidence that planning of musical sequences was more disrupted by incongruent action-perception mappings for musicians than for nonmusicians. However, their study focused on reaction time rather than error rates and may be more relevant to the way plans are generated (preplanning) than to how they are implemented during a performance (P. Keller, personal communication, July 26, 2004).

One subtle difference between groups with respect to patterns of disruption from altered feedback concerns the influence of randomized feedback pitch. Whereas these conditions led to baseline levels of error rates for nonpianists (see Figures 4 and 7), they caused slightly higher error rates for pianists (see Figures 2 and 6). This led to a null difference between errors associated with lag-1 and randomized feedback for pianists in Experiment 1, whereas the same pair differed significantly for nonpianists in Experiment 2. Similarly, pianists in Experiment 3 demonstrated slightly greater disruption from random-same conditions than did nonpianists in Experiment 4 . These results suggest that pianists are more sensitive to any kind of mismatch between an action and the expected consequent pitch, whereas nonpianists can overcome many mismatches unless the nonmatching pitch is associated with an event intended for another sequence position. Thus, learned action- effect associations may influence the way pianists respond to individual pitch events, whereas the relation between movements from one key to the other and the consequent melodic trajectory are less influenced by acquired skill. In keeping with this, eventrelated potential studies have shown that trained musicians respond to individual tones with enhanced auditory evoked potentials in comparison with nonmusicians (Shahin, Bosnyak, Trainor, \& Roberts, 2003), whereas both groups respond similarly to changes in melodic contour (Trainor, Desjardins, \& Rockel, 1999).

\section{Conclusions}

The current experiments demonstrate that performers with and without musical training are sensitive to the relation between the melodic structure of a sequence that they produce and the sequence of feedback events that results from their actions. Experiments 1 and 2 confirm the results of past experiments (Finney, 1997; Finney \& Palmer, 2003; Pfordresher, 2003a; Pfordresher \& Palmer, in press; Repp, 1999): Disruption occurred when pitches repeated previously planned events but not when pitches were selected randomly or were absent. Experiments 3-5 further explore similarity between produced and perceived sequences to demonstrate that disruption scales with similarity, defined at multiple hierarchical levels between produced and perceived sequences. Overall, results are consistent with the proposal that perception and action share a common representation and that interference of production happens when the events in the feedback sequence are associated with events planned for production at other positions. Musical training did not qualify these results; hence, effects of perception-action relations during musical performance cannot result merely from the acquisition of specialized skills.

\section{References}

Adams, J. A. (1971). A closed-loop theory of motor learning. Journal of Motor Behavior, 3, 111-149.

Bangert, M., \& Altenmüller, E. O. (2003). Mapping perception to action in piano practice: A longitudinal DC-EEG study. BMC Neuroscience, 4, $26-40$.

Bjork, E. L., \& Healy, A. F. (1974). Short-term order and item retention. Journal of Verbal Learning and Verbal Behavior, 13, 80-97.

Black, J. W. (1951). The effect of delayed side-tone upon vocal rate and intensity. Journal of Speech and Hearing Disorders, 16, 56-60.

Boltz, M., \& Jones, M. R. (1986). Does rule recursion make melodies easier to reproduce? If not, what does? Cognitive Psychology, 18, $389-431$.

Chase, R. A. (1965). An information-flow model of the organization of motor activity: I. Transduction, transmission and central control of sensory information. Journal of Nervous and Mental Disease, 140, $239-251$.

Cho, Y. S., \& Proctor, R. W. (2003). Stimulus and response representations underlying orthogonal stimulus-response compatibility effects. Psychonomic Bulletin \& Review, 10, 45-73.

Chomsky, N. (1957). Syntactic structures. The Hague, the Netherlands: Mouton.

Collard, R., \& Povel, D. J. (1982). Theory of serial pattern production: Tree traversals. Psychological Review, 89, 693-707.

Dell, G. S. (1986). A spreading activation theory of retrieval in language production. Psychological Review, 93, 293-321.

Dowling, W. J. (1978). Scale and contour: Two components of a theory of memory for melodies. Psychological Review, 85, 341-354.

Drake, C., Jones, M. R., \& Baruch, C. (2000). The development of 
rhythmic attending in auditory sequences: Attunement, referent period, focal attending. Cognition, 77, 251-288.

Drake, C., \& Palmer, C. (2000). Skill acquisition in music performance: Relations between planning and temporal control. Cognition, 74, 1-32.

Drake, C., Penel, A., \& Bigand, E. (2000). Tapping in time with mechanically and expressively performed music. Music Perception, 18, 1-24.

Drost, U., Rieger, M., Brass, M., Gunter, T., \& Prinz, W. (2005). Actioneffect coupling in pianists. Psychological Research, 69, 233-241.

Elsner, B., \& Hommel, B. (2001). Effect anticipation and action control. Journal of Experimental Psychology: Human Perception and Performance, 27, 229-240.

Ericsson, K. A., \& Charness, N. (1994). Expert performance. American Psychologist, 49, 725-747.

Fairbanks, G. (1954). Systematic research in experimental phonetics: 1. A theory of the speech mechanism as a servosystem. Journal of Speech and Hearing Disorders, 19, 133-139.

Fairbanks, G., \& Guttman, N. (1958). Effects of delayed auditory feedback upon articulation. Journal of Speech and Hearing Research, 1, 333-346.

Finney, S. A. (1997). Auditory feedback and musical keyboard performance. Music Perception, 15, 153-174.

Finney, S. A. (2001). FTAP: A Linux-based program for tapping and music experiments. Behavior Research Methods, Instruments, \& Computers, $33,65-72$.

Finney, S. A., \& Palmer, C. (2003). Auditory feedback and memory for music performance: Sound evidence for an encoding effect. Memory \& Cognition, 31, 51-64.

Finney, S. A., \& Warren, W. H. (2002). Delayed auditory feedback and rhythmic tapping: Evidence for a critical interval shift. Perception \& Psychophysics, 64, 896-908.

Gates, A., \& Bradshaw, J. L. (1974). Effects of auditory feedback on a musical performance task. Perception \& Psychophysics, 16, 105-109.

Greenwald, A. G. (1970). Sensory feedback mechanisms in performance control: With special reference to the ideo-motor mechanism. Psychological Review, 77, 73-99.

Gupta, P., \& Cohen, N. J. (2002). Theoretical and computational analysis of skill learning, repetition priming, and procedural memory. Psychological Review, 109, 401-448.

Haslinger, B., Erhard, P., Altenmüller, E. O., Hettenlotter, A., Schwaiger, M., von Einsiedel, H. G., et al. (2004). Reduced recruitment of motor association areas during bimanual coordination in concert pianists. $\mathrm{Hu}$ man Brain Mapping, 22, 206-215.

Havlicek, L. (1968). Effects of delayed auditory feedback on musical performance. Journal of Research in Music Education, 16, 308-318.

Healy, A. F. (1974). Separating item from order information in short-term memory. Journal of Verbal Learning and Verbal Behavior, 13, 644655.

Hommel, B., Müsseler, J., Aschersleben, G., \& Prinz, W. (2001). The theory of event coding (TEC): A framework for perception and action planning. Behavioral and Brain Sciences, 24, 849-937.

Hommel, B., \& Prinz, W. (Eds.). (1997). Theoretical issues in stimulusresponse compatibility. Amsterdam: North-Holland.

Howell, P. (2001). A model of timing interference to speech control in normal and altered listening conditions applied to the treatment of stuttering. In B. Maassen, W. Hulstijn, R. Kent, H. F. M. Peters, \& P. H. M. M. van Lieshout (Eds.), Speech motor control in normal and disordered speech (pp. 291-294). Nijmegen, the Netherlands: Uttgeverij Vantilt.

Howell, P. (2004). Assessment of some contemporary theories of stuttering that apply to spontaneous speech. Contemporary Issues in Communicative Sciences and Disorders, 39, 122-139.

Howell, P., \& Archer, A. (1984). Susceptibility to the effects of delayed auditory feedback. Perception \& Psychophysics, 36, 296-302.

Howell, P., \& Au-Yeung, J. (2002). The EXPLAN theory of fluency control and the diagnosis of stuttering. In E. Fava (Ed.), Pathology and therapy of speech disorders (pp. 75-94). Amsterdam: John Benjamins.

Howell, P., Powell, D. J., \& Khan, I. (1983). Amplitude contour of the delayed signal and interference in delayed auditory feedback tasks. Journal of Experimental Psychology: Human Perception and Performance, 9, 772-784.

Jones, M. R. (1976). Time, our lost dimension: Toward a new theory of perception, attention, and memory. Psychological Review, 83, 323-355.

Jones, M. R. (1987). Dynamic pattern structure in music: Recent theory and research. Perception \& Psychophysics, 41, 621-634.

Jones, M. R. (1990). Learning and the development of expectancies: An interactionist approach. Psychomusicology, 9, 193-228.

Jones, M. R., \& Pfordresher, P. Q. (1997). Tracking melodic events using joint accent structure. Canadian Journal of Experimental Psychology, $51,271-291$.

Keele, S. W., Ivry, R., Mayr, U., Hazeltine, E., \& Heuer, H. (2003). The cognitive and neural architecture of sequence representation. Psychological Review, 110, 316-339.

Keller, P., \& Koch, I. (2005). Action planning in sequential skills: Relations to music performance. Manuscript submitted for publication.

Keppel, G. (1991). Design and analysis: A researcher's handbook. Upper Saddle River, NJ: Prentice Hall.

Krumhansl, C. L. (1990). Cognitive foundations of musical pitch. New York: Oxford University Press.

Krumhansl, C. L. (2000). Rhythm and pitch in music cognition. Psychological Bulletin, 126, 159-179.

Kunde, W. (2001). Response-effect compatibility in manual choice reaction tasks. Journal of Experimental Psychology: Human Perception and Performance, 27, 387-394.

Kunde, W., Koch, I., \& Hoffmann, J. (2004). Anticipated action effects affect the selection, initiation, and execution of actions. Quarterly Journal of Experimental Psychology: Human Experimental Psychology, 57(A), 87-106.

Large, E. W. (1993). Dynamic programming for the analysis of serial behaviors. Behavior Research Methods, Instruments, \& Computers, 25, $238-241$.

Lashley, K. (1951). The problem of serial order in behavior. In L. A. Jeffress (Ed.), Cerebral mechanisms in behavior (pp. 112-136). New York: Wiley.

Lee, B. S. (1950). Effects of delayed speech feedback. Journal of the Acoustical Society of America, 22, 824-826.

Lerdahl, F., \& Jackendoff, R. (1983). A generative theory of tonal music. Cambridge, MA: MIT Press.

Levitin, D. J., \& Menon, V. (2003). Musical structure is processed in "language" areas of the brain: A possible role for Brodmann Area 47 in temporal coherence. NeuroImage, 20, 2142-2152.

MacKay, D. G. (1987). The organization of perception and action. New York: Springer-Verlag.

Martin, J. G. (1972). Rhythmic (hierarchical) versus serial structure in speech and other behavior. Psychological Review, 79, 485-509.

Müsseler, J. (1999). How independent from action control is perception? An event coding account for more equally-ranked crosstalks. In G. Aschersleben, T. Bachmann, \& J. Müsseler (Eds.), Cognitive contributions to the perception of spatial and temporal events (pp. 121-148). Amsterdam: Elsevier.

Palmer, C., \& Drake, C. (1997). Monitoring and planning capacities in the acquisition of music performance skills. Canadian Journal of Experimental Psychology, 51, 369-384.

Palmer, C., \& Pfordresher, P. Q. (2003). Incremental planning in sequence production. Psychological Review, 110, 683-712.

Palmer, C., \& van de Sande, C. (1993). Units of knowledge in music performance. Journal of Experimental Psychology: Learning, Memory, and Cognition, 19, 457-470.

Palmer, C., \& van de Sande, C. (1995). Range of planning in skilled music 
performance. Journal of Experimental Psychology: Human Perception and Performance, 21, 947-962.

Pfordresher, P. Q. (2003a). Auditory feedback in music performance: Evidence for a dissociation of sequencing and timing. Journal of Experimental Psychology: Human Perception and Performance, 29, 949964

Pfordresher, P. Q. (2003b). The role of melodic and rhythmic accents in musical structure. Music Perception, 20, 431-464.

Pfordresher, P. Q. (2005). Melodic trajectories and movement trajectories in action sequences. Manuscript in preparation.

Pfordresher, P. Q., \& Palmer, C. (in press). Effects of hearing the past, present, or future during music performance. Perception and Psychophysics.

Pinker, S. (1997). How the mind works. New York: Norton.

Prinz, W. (1997). Perception and action planning. European Journal of Cognitive Psychology, 9, 129-154.

Repp, B. H. (1995). Detectability of duration and intensity increments in melody tones: A partial connection between music perception and performance. Perception \& Psychophysics, 57, 1217-1232.

Repp, B. H. (1998a). Obligatory "expectations" of expressive timing induced by perception of musical structure. Psychological Research, 61, 33-43.

Repp, B. H. (1998b). Variations on a theme by Chopin: Relations between perception and production of timing in music. Journal of Experimental Psychology: Human Perception and Performance, 24, 791-811.

Repp, B. H. (1999). Effects of auditory feedback deprivation on expressive piano performance. Music Perception, 16, 409-438.

Rosenbaum, D. A., Kenny, S. B., \& Derr, M. A. (1983). Hierarchical control of rapid movement sequences, Journal of Experimental Psychology: Human Perception and Performance, 9, 86-102.
Schlaug, G., Jäncke, L., Huang, Y., \& Steinmetz, H. (1995, February 3). In vivo evidence of structural brain asymmetry in musicians. Science, 267, 699-700.

Shahin, A., Bosnyak, D. J., Trainor, L. J., \& Roberts, L. E. (2003). Enhancement of neuroplastic P2 and N1c auditory evoked potentials in musicians. Journal of Neuroscience, 23, 5545-5552.

Stevens, L. T. (1886). On the time sense. Mind, 11, 393-404.

Stöcker, C., Sebald, A., \& Hoffman, J. (2003). The influence of responseeffect compatibility in a serial reaction time task. Quarterly Journal of Experimental Psychology: Human Experimental Psychology, 56(A), 685-703.

Trainor, L. J., Desjardins, N., \& Rockel, C. (1999). A comparison of contour and interval processing in musicians and nonmusicians using event-related potentials. Australian Journal of Psychology, 51, 147-153.

Vorberg, D., \& Wing, A. (1996). Modeling variability and dependence in timing. In H. Heurer \& S. W. Keele (Eds.), Handbook of perception and action (pp. 181-262). London: Academic Press.

Wallin, N. L., Merker, B., \& Brown, S. (2001). The origins of music. Cambridge, MA: MIT Press.

Wiener, N. (1948). Cybernetics. Cambridge, MA: MIT Press.

Wing, A. M., \& Kristofferson, A. B. (1973). The timing of interresponse intervals. Perception \& Psychophysics, 13, 455-460.

Zatorre, R. J., \& Peretz, I. (2001). The biological foundations of music. Annals of the New York Academy of Sciences, 930, 232-258.

Received September 27, 2004

Revision received April 29, 2005

Accepted May 5, 2005 